\title{
Satisfaction Assessment of Telephone Consultations among 270 Primary Health Care Physicians During the COVID-19 Pandemic in Qatar
}

\author{
Moqthiar Sharief*, Maxwell Sleiman, Samina Jabeen and Shawqiya Alamjid \\ Primary Health Care Corporation (PHCC), Doha P.O. Box 26555, Qatar
}

\begin{abstract}
Background: The COVID-19 outbreak has placed the Health Service under significant strain worldwide. In Qatar, health care authorities (i.e., the primary Health Care Corporation (PHCC) which is one of the main governmental health care facilities in Qatar) has rapidly adopted telemedicine solutions as an alternative to face-to-face consultations during the COVID-19 pandemic. Prior to the current pandemic, the majority of healthcare professionals were unfamiliar with Telemedicine. Additionally, remote consultation is projected to continue for the foreseeable future; consequently, this study sought to determine physician satisfaction with telephone consultation utilization.
\end{abstract}

Methods: Amongst, 724 physicians enrolled to this study, 270 responded to the survey and were included in the study. All the physicians were part of PHCC and belongs to different Health Centres. The survey was sent via official institution email (Email) to all the registered physicians and was completed between the $13^{\text {th }}$ of September 2020 and the of $15^{\text {th }}$ March 2021.

Results: The majority of physicians surveyed in this study expressed high satisfaction with telephone consultations. Over $70 \%$ of physicians surveyed stated that telemedicine services increased their job efficacy and performance, offered them greater control over their work, enabled them to complete jobs more rapidly, and allowed them to be more productive. Additionally, they stated that they felt secure using telemedicine services during the COVID-19 pandemic.

Conclusion: To ensure that health services are always meeting patients' requirements, flexibility in care delivery modes, role shifting to accommodate virtual care, sustained relationships with patients, and interdisciplinary collaboration are required. These efforts will be successful only with clear rules and training, as well as thorough consideration of technological barriers and human interaction requirements. Beyond COVID-19, other activities are required to ensure clinically suitable and acceptable virtual consultations.

KEYWORDS: Patient Satisfaction; Telemedicine; Pandemic; Health service

\section{INTRODUCTION}

With over 270 million people worldwide infected with COVID-19 and over 5,3 million deaths since the pandemic began [1] the World Health Organization (WHO) has recommended the implementation of public health measures such as the quarantine of all individuals suspected of infection for 14 days [2-5]. Numerous countries have also implemented "social distancing" and "lock-downs" of varying degrees of severity for entire communities in an effort to contain the spread of COVID-19 [6-15].
Quick Response Code: (PHCC), Doha P.O. Box 26555, Qatar

Received: November 15, 2021 Reviewed: December 8, 2021 Published: December 23, 2021

How to cite this article: Moqthiar S, Maxwell S, Samina J, Shawqiya A. Satisfaction Assessment of Telephone Consultations among 270 Primary Health Care Physicians During the COVID-19 Pandemic in Qatar. 2021- 3(6) OAJBS.ID.000363. DOI: 10.38125/OAJBS.000363 
Since the pandemic's inception [16,17], telemedicine has enabled remote triage of patients, rapid access to information, routine follow-up treatment (particularly important for managing chronic diseases), remote diagnosis, and remote management of patients since the pandemic's inception $[16,17]$. This method of care delivery has aided in the reduction of demand for emergency services and increased access for some patients $[18,19]$. Additional benefits have been noted, including convenience, cost savings, and the simplicity with which interdisciplinary visits and real-time consultations can be organized. However, research has emphasized the importance of sustaining meaningful teamwork [20-22].

The downsides of telemedicine that have been found thus far include the possibility for therapeutic connections to deteriorate and diminished continuity of care, as well as a lack of psychosocial support and depersonalization of practice [20,23,24]. Other identified disadvantages of telemedicine include the risk of compromised confidentiality and patients' unequal access to and capacity for using technology, which may result in certain populations being excluded from this type of care, including the elderly $[20,22,23]$ and vulnerable populations (e.g., those living in rural areas or on a low income, ethnic minorities, and allophones) [25-27]. Another disadvantage of telemedicine is the inability to conduct physical examinations directly [20]. Finally, concerns have been raised about the compatibility of some professional tasks with telemedicine, as well as interprofessional cooperation challenges (workload, isolation, and a lack of socialization time) [22]

The requirement of delivering telemedicine that is responsive to the needs of patients during a pandemic has created numerous obstacles for providers of primary health care. Its rapid use has sparked concerns regarding the ramifications of its use in a variety of clinical settings. Physicians accelerated their adoption of telemedicine in response to the COVID-19 epidemic, although data on physician attitudes towards telemedicine is limited [28]. To our knowledge, little research has examined physicians' perceptions of rapid telemedicine implementation in the context of the COVID-19 outbreak.

Thus, the aim of the present study was to (i) project physicians total rate of satisfaction with teleconsultation as well as various perspectives of teleconsultation like the benefits to practice, such as the ease of use, workflow, communication, and productivity, and (ii) measure the attitudes toward the future use of telemedicine in Qatar.

\section{METHODOLOGY}

\section{Research Design}

The design of this research was chosen to be mixed as it comprises primary as well as secondary methods of collecting information. The primary research designs help the research gain information directly from the perspective of a large number of people, as well as ensure that the research is known to be valid, authentic, and reliable. On the other hand, the secondary research design is used in the research so that the study can refer to the research that has been conducted previously by other researchers. This allows the present research to find the literature gaps and review the same topic from multiple perspectives.

\section{Research Approach}

The approach used for the mixed research design is quantitative. It is a procedure of gathering information and analysing the numerical information. This can help the study find the trends, patterns, and behaviours of the topic being researched. The quantitative research will enhance the study by determining statistical information about the topic through surveys.

\section{Study Setting}

The study was conducted in primary health care centres in Qatar, which is the premier institute serving all the populations of Qatar catering to their primary health care needs. We have 724 physicians who work in PHCC Health Centres undertaking various services, including general clinics, antenatal services, and wellbaby clinics, to name a few. Since the COVID pandemic, a need has arisen to safeguard patients and the healthcare workforce, and teleconsultation services have been implemented in all PHCC health centres. The study was conducted amongst physicians undertaking teleconsultation services in PHCC Qatar.

\section{Population}

The registered physicians working in the PHC centres in Qatar are the participants chosen for the survey questionnaire.

\section{Sample size Calculation}

There are 724 physicians working in PHCCs. The vast majority of those were eligible for the study since during the COVID-19 pandemic, most consultations in PHCC were done using telemedicine and, in only specific cases, in-person consultation was allowed. Although there is no need for sample size justification as this study is using a convenient sample, we are showing the below justification to enable the reviewers to have an idea about the estimated precision coming from this study. Assuming that $10 \%$ of the physicians were not eligible and a response rate of $40 \%$, the study with $\mathrm{N}=260$ will be able to assess the prevalence of satisfaction to within a margin of error of at most $5 \%$ using a $95 \%$ confidence interval. A lower response rate of $25 \%$ will result in a slight increase in the margin of error to at most $8 \%$.

\section{Data Collection}

A structured questionnaire, based on the literature and the objectives of the study, was developed in English since all PHCC physicians are English speakers. This questionnaire was sent via official PHCC email to all the registered physicians who are undertaking telephone consultations in PHCC Qatar. The questionnaire includes:

1- Demographics section with age, gender, and marital status

2- Work relations questions such as rank, language spoken to patients, years of experience, years of experience in Qatar, prior experience of telephone consultation with patients, prior training on telephone consultation with patients

3- Questions, adopted from the literature, related to ease of use, usefulness, and satisfaction.

4- Questions related to the willingness of physicians to continue using the services even under normal circumstances.

\section{Types of Outcome Measurements}

Most of the variables are categorical, such as gender, prior training (yes vs. no), and rank of the physician. The main outcome of the answers to scale questions is measured on a 5-point Likert scale, from strongly agree to strongly disagree. Some variables, 
such as age and years of experience, are either numeric or grouped into categories.

\section{Ethical Approval Statement}

This was a cross-sectional experimental study. The study protocol was in accordance with the Helsinki Declaration for conducting human experimentation and was approved by the ethical committee of the primary health care corporation (PHCC) (PHCC/DCR/2020/07/084). All participants have signed an informed consent after receiving a complete verbal description of the protocol.

\section{RESULTS}

\section{Participants}

Table 1: Demographic information of the Physicians enrolled to the study ( $N=270)$.

\begin{tabular}{|c|c|c|c|}
\hline Characteristics & Categories & Number (n) & Percentage (\%) \\
\hline \multirow{5}{*}{ AGE } & $25-34$ & 21 & $8.50 \%$ \\
\hline & $35-44$ & 94 & $37.90 \%$ \\
\hline & $45-54$ & 92 & $37.10 \%$ \\
\hline & $55-64$ & 35 & $14.10 \%$ \\
\hline & $65+$ & 6 & $2.40 \%$ \\
\hline \multirow{2}{*}{ Gender } & Female & 105 & $42.50 \%$ \\
\hline & Male & 142 & $57.50 \%$ \\
\hline \multirow{3}{*}{ Marital Status } & Divorced/ Separated / Widowed & 8 & $3.30 \%$ \\
\hline & Married & 198 & $80.50 \%$ \\
\hline & Single & 40 & $16.30 \%$ \\
\hline \multirow{3}{*}{ Do you have any children? } & No & 59 & $24.00 \%$ \\
\hline & Yes, all 18 or over & 61 & $24.80 \%$ \\
\hline & Yes, one or more under 18 & 126 & $51.20 \%$ \\
\hline \multirow{2}{*}{ Currently living with family? } & No & 78 & $31.80 \%$ \\
\hline & Yes & 167 & $68.20 \%$ \\
\hline \multirow{8}{*}{$\begin{array}{l}\text { Which of the following best } \\
\text { describes your current occupation? }\end{array}$} & Resident & 13 & $5.20 \%$ \\
\hline & Fellow & 7 & $2.80 \%$ \\
\hline & General Practitioner & 90 & $36.30 \%$ \\
\hline & Physician Lead & 1 & $0.40 \%$ \\
\hline & Specialist & 14 & $5.60 \%$ \\
\hline & Consultant & 97 & $39.10 \%$ \\
\hline & Senior Consultant & 19 & $7.70 \%$ \\
\hline & Manager & 7 & $2.80 \%$ \\
\hline \multirow{2}{*}{ Place of Work } & PHCC Headquarters & 33 & $13.50 \%$ \\
\hline & PHCC Health Centre & 211 & $86.50 \%$ \\
\hline
\end{tabular}

Two hundred seventy physicians participated in the study (13 residents, 7 fellows, 90 general practitioners, 1 physician lead, 14 specialists, 97 consultants, 19 senior consultants, and 7 managers). Table 1 shows the demographic information of the physicians who participated in the study.

\section{Survey Outcomes}

Most physicians in this study reported high levels of satisfaction with telephone consultations. Over $70 \%$ of the physician enrolled to this study mentioned that telemedicine services improved their job effectiveness and performance, gave them greater control over their work, allowed them to accomplish their tasks quickly, to be more productive. And mentioned that they were comfortable using telemedicine service during COVID-19 pandemic. Details of the satisfaction survey are presented in Table 2 .

\section{DISCUSSION}

As the COVID-19 pandemic engulfed the world and healthcare systems worldwide crumbled, the Qatar Ministry of Public Health, in collaboration with the HMC and PHCC, pioneered the use of teleconsultation services for Qatari citizens and residents in order to reduce contact transmission and cross infection between patients and healthcare providers.

The majority of physicians surveyed in this study expressed high satisfaction with telephone consultations. Indeed, our findings on both positive and negative attitudes toward telemedicine are consistent with the post-COVID-19 literature [20-22,28]. In keeping with the findings of a recent California study, physicians in our study believed telemedicine had the potential to improve health care access, is well-suited for treating a number of medical disorders, and can enhance patient care in a variety of ways [28]. 
Table 2: Satisfaction survey amongst 270 primary care physicians.

\begin{tabular}{|c|c|c|c|c|c|c|c|c|c|c|}
\hline \multirow[t]{2}{*}{ Survey Parameters } & \multicolumn{2}{|c|}{ Strongly Agree } & \multicolumn{2}{|c|}{ Agree } & \multicolumn{2}{|c|}{$\begin{array}{c}\text { Neither Agree } \\
\text { nor Disagree }\end{array}$} & \multicolumn{2}{|c|}{ Disagree } & \multicolumn{2}{|c|}{$\begin{array}{l}\text { Strongly } \\
\text { Disagree }\end{array}$} \\
\hline & $\mathbf{N}$ & $\%$ & $\mathbf{N}$ & $\%$ & $\mathbf{N}$ & $\%$ & $\mathbf{N}$ & $\%$ & $\mathbf{N}$ & $\%$ \\
\hline $\begin{array}{l}\text { Using telemedicine services improves my job } \\
\text { effectiveness and performance }\end{array}$ & 52 & $21.10 \%$ & 119 & $48.20 \%$ & 60 & $24.30 \%$ & 12 & $4.90 \%$ & 4 & $1.60 \%$ \\
\hline $\begin{array}{l}\text { Using Telemedicine services gives me greater } \\
\text { control over my work }\end{array}$ & 61 & $24.60 \%$ & 125 & $50.40 \%$ & 44 & $17.70 \%$ & 17 & $6.90 \%$ & 1 & 0.4 \\
\hline $\begin{array}{l}\text { Telemedicine allows me to accomplish my tasks } \\
\text { quickly }\end{array}$ & 65 & $26.30 \%$ & 130 & $52.60 \%$ & 40 & $16.20 \%$ & 11 & $4.50 \%$ & 1 & $0.40 \%$ \\
\hline Telemedicine enables me to be productive & 65 & $26.20 \%$ & 126 & $50.80 \%$ & 42 & $16.90 \%$ & 12 & $4.80 \%$ & 3 & $1.20 \%$ \\
\hline Telemedicine user is friendly & 79 & $31.90 \%$ & 133 & $53.60 \%$ & 31 & $12.50 \%$ & 5 & $2.00 \%$ & 0 & $0.00 \%$ \\
\hline $\begin{array}{l}\text { Integrating with patients via Telemedicine is } \\
\text { often frustrating }\end{array}$ & 43 & $17.50 \%$ & 72 & $29.30 \%$ & 58 & $23.60 \%$ & 61 & $24.80 \%$ & 12 & $4.90 \%$ \\
\hline $\begin{array}{l}\text { Telemedicine service does not require multiple } \\
\text { formal training session to be effective }\end{array}$ & 56 & $22.60 \%$ & 130 & $52.40 \%$ & 40 & $16.10 \%$ & 18 & $7.30 \%$ & 4 & $1.60 \%$ \\
\hline $\begin{array}{l}\text { Telemedicine service is compatible with current } \\
\text { clinical workflow }\end{array}$ & 61 & $24.80 \%$ & 139 & $56.50 \%$ & 31 & $12.60 \%$ & 13 & $5.30 \%$ & 2 & $0.80 \%$ \\
\hline $\begin{array}{l}\text { I have a positive opinion about use of } \\
\text { telemedicine service }\end{array}$ & 80 & $32.40 \%$ & 129 & $52.20 \%$ & 27 & $10.90 \%$ & 10 & $4.00 \%$ & 1 & $0.40 \%$ \\
\hline $\begin{array}{l}\text { Telemedicine service positively enables me } \\
\text { to devise treatment strategies for my patient } \\
\text { without }\end{array}$ & 70 & $28.30 \%$ & 131 & $53.00 \%$ & 31 & $12.60 \%$ & 14 & $5.70 \%$ & 1 & $0.40 \%$ \\
\hline $\begin{array}{l}\text { telemedicine service enables me to provide a } \\
\text { more comprehensive care for my patients }\end{array}$ & 57 & $23.20 \%$ & 123 & $50.00 \%$ & 41 & $16.70 \%$ & 23 & $9.30 \%$ & 2 & $0.80 \%$ \\
\hline $\begin{array}{l}\text { I have gained rich and diverse experience in } \\
\text { telemedicine service delivery }\end{array}$ & 65 & $26.40 \%$ & 142 & $57.70 \%$ & 33 & $13.40 \%$ & 4 & $1.60 \%$ & 2 & $0.80 \%$ \\
\hline $\begin{array}{l}\text { Telemedicine service is beneficial for my } \\
\text { patients during the COVID-19 pandemic }\end{array}$ & 107 & $43.50 \%$ & 127 & $51.60 \%$ & 12 & $4.90 \%$ & 0 & $0.00 \%$ & 0 & $0.00 \%$ \\
\hline $\begin{array}{l}\text { Telemedicine service enables better use of } \\
\text { physicians' time in treating patients during } \\
\text { COVID-19 }\end{array}$ & 99 & $40.40 \%$ & 124 & $50.60 \%$ & 16 & $6.50 \%$ & 4 & $1.60 \%$ & 2 & $0.80 \%$ \\
\hline $\begin{array}{l}\text { Telemedicine service has enabled safely for both } \\
\text { patients and physicians during the COVID }\end{array}$ & 108 & $44.10 \%$ & 120 & $49.00 \%$ & 14 & $5.70 \%$ & 2 & $0.80 \%$ & 1 & $0.40 \%$ \\
\hline $\begin{array}{l}\text { I feel comfortable using telemedicine service } \\
\text { during COVID-19 pandemic }\end{array}$ & 99 & $39.90 \%$ & 126 & $50.80 \%$ & 23 & $9.30 \%$ & 0 & $0.00 \%$ & 0 & $0.00 \%$ \\
\hline $\begin{array}{l}\text { I am satisfied with telemedicine service delivery } \\
\text { during COVID-19 pandemic and wish to add }\end{array}$ & 88 & $36.10 \%$ & 124 & $50.80 \%$ & 21 & $8.60 \%$ & 11 & $4.50 \%$ & 0 & $0.00 \%$ \\
\hline $\begin{array}{l}\text { Overall physicians are satisfied with use of } \\
\text { telemedicine service during covid-19 pandemic }\end{array}$ & 74 & $30.00 \%$ & 142 & $57.50 \%$ & 27 & $10.90 \%$ & 3 & $1.20 \%$ & 1 & $0.40 \%$ \\
\hline
\end{tabular}

As the use of telemedicine grows concurrently with the continued use of face-to-face consultations, it is critical to develop measures to guarantee that this mode of care delivery is secure and equitable in both routine and emergency settings [20,21,23]. Our findings emphasize the benefits of telemedicine for physicians, including the convenience and flexibility of working from home, schedule flexibility, increased availability for their patients, and the assurance of remuneration for telemedicine visits. Prior to COVID-19, the primary impediments to telemedicine development were payment constraints and organizational issues within the health system [16]. Prior to the pandemic, physicians in Quebec's public sector $(70$ percent of primary care physicians and 82 percent of specialists in 2015) were compensated on a fee-for-service basis $[29,30]$, but only specialist physicians were compensated for telemedicine [30-32], which primarily consisted of follow-up telephone calls. Prior to the pandemic, physicians in the United States did not use telemedicine due to worries about funding, medico-legal risks, potential inefficiencies, sluggish acceptance of technical tools, and a lack of telemedicine training for healthcare professionals [33,34].
Our findings indicate that physicians like the fact that telemedicine is now recognized as a formal mode of care provision for which physicians can be compensated in the PHCC. Notably, the PHCC in Qatar implemented interim measures to eliminate this barrier during the epidemic and is now working to make them permanent.

Previously, clinicians' hesitation to utilize telemedicine was due in part to their negative impressions of this tool $[16,35]$. Due to the intricacy of telemedicine, the considerable changes it brought to the way healthcare professionals work, and the belief that it was not instantly effective, safe, or even prevalent, many doctors chose not to use it [16].

According to previous findings, physicians who practice telemedicine have more nuanced opinions on the prospects for reliable diagnoses via telemedicine in a variety of situations. A physical examination is not always necessary, and the patient's history may suffice to make a diagnosis. On the other hand, it has been observed that diagnosis without a physical examination and visual 
information might be extremely difficult in some circumstances, posing the danger of error. Thus, a recent study demonstrated that this inability to conduct direct physical examinations can be partially mitigated by involving patients in reporting their own data (e.g., temperature) and by utilizing video consultations to allow providers to observe patients' general appearance and symptoms (e.g., breathing, coughing) [17]. Additionally, providers have rediscovered the value of taking a thorough medical history and honing their observation abilities in order to make a diagnosis [21]. Additionally, our respondents emphasized the importance of developing criteria for determining which instances are best handled by telephone, video, or in person.

A further challenge in crisis situations, like the COVID-19 pandemic, is the requirement for rapid adoption of novel consultation approaches $[16,35]$. Our study established a strong necessity for providers to adapt their responsibilities and practices. This is congruent with the findings of a previous study [16], which highlighted the compatibility or incompatibility of certain professional responsibilities with the delivery of patient care via telemedicine. Additionally, some professionals took on additional responsibilities and were forced to adapt to or assume new roles to assist with crisis management in their clinics, which corroborated our findings above about the need to replace nurses or administrative assistants in telephone triage during the pandemic.

In terms of efficiency, training may be beneficial in addressing the diagnostic and administrative issues that clinicians confront More experienced medical professionals appear to be more adept at picking up telemedicine abilities, which should be considered when training future practitioners who will have more experience with telemedicine. Medical education is critical in reinforcing and honing the observational abilities required to obtain a diagnosis remotely [21]. To deliver telemedicine effectively during a public health emergency like the COVID-19 pandemic, experts must be trained and prepared to employ a variety of consultation methods while also responding to the needs of individual patients. However reserving telemedicine use for crises would be detrimental to its progress and safety following the crisis [16]. Our respondents remarks about the lack of rules for telemedicine use focused mostly on the negative consequences of not emphasizing the social and emotional components of care delivery alongside the medical dimensions of care that are critical in primary health care.

A critical role shift for providers entails conducting a physical examination during a virtual appointment. Ourrespondents believed that the physical examination is not only necessary for good clinical practice, but also a necessary component of the physician's function and a basic element of the therapeutic interaction with the patient They noticed that establishing this relationship only through telemedicine was challenging, particularly when the physician had no prior interaction with the patient. Additionally, studies have emphasized the possibility that telemedicine modalities may jeopardize the therapeutic relationship and continuity of care, both of which are critical components of clinical practice and have substantial implications for both patients and clinicians $[20,23,24]$ Effective treatment of mental, emotional, and behavioral health disorders requires a strong patient-provider relationship [24]. Humanism should be fundamental in the context of virtual care [23]. Providers' experiences during the current COVID-19 pandemic have emphasized the need of remote social links for curative human relationships in addition to guaranteeing the safety and efficiency of care delivery [24]. Simultaneously, promoting continuity of care and building therapeutic interactions with patients in a virtual care setting requires the development of novel methods for initiating meaningful partnerships via personal and situational behaviors [24].

Additionally, our findings imply that technical impediments should not be overlooked, as they can impact both patients' access to care and physicians' ability to give high-quality care. This is consistent with research indicating that some patients and providers struggle with technological literacy and logistical barriers associated with participating in telemedicine visits, particularly given the variety of available technologies and/or the manner in which some medical practices have shifted technologies [20-22]. Our findings indicate that these impediments may cause telemedicine users to revert to audio-only telephone consultations, highlighting the need to resolve these technological challenges.

\section{LIMITATIONS}

We note that our findings are based on participants' impressions at a particular point in the health crisis and do not reflect the overall experience in primary health care practice, a situation that is still unfolding. Subsequent research might examine physicians' and other stakeholders' perceptions of telemedicine in primary health care practice on a periodic basis to describe and understand how its effects vary over time. Future research into the long-term implications of telemedicine use following a pandemic will help us better grasp the role of telemedicine in primary healthcare delivery. Additionally, future studies examining patients' perceptions of telemedicine and tracking those perceptions through time would provide significant knowledge.

\section{CONCLUSION}

The COVID-19 pandemic and the likelihood of additional outbreaks have established beyond a shadow of a doubt that telemedicine is here to stay. Health Care Professionals reported adopting novel ways with little training but with a positive perception in general. Telemedicine can be used to treat some categories of patients in the post-COVID-19 age, but the physician operating these clinics must have the necessary skills and knowledge. To guarantee that telemedicine care delivery satisfies the needs of both patients and clinicians, it will be necessary to support the deployment of telemedicine, provide professional standards and training, and pay particular attention to both technological barriers and human interaction needs. Addressing these concerns, we believe, can help alleviate hurdles and promote the introduction of safe and effective virtual care.

\section{DECLARATIONS}

\section{Availability of Data and Material}

All data analysed and reported in this study are available from the first author on reasonable request.

\section{Consent to Participate}

All participants provided informed consent before participating in the study.

\section{Consent for Publication}

All Physicians provided consent for anonymous data use for research purposes and publications. All authors approved of the final version to be published and agree to be accountable for any part of the work. 


\section{REFERENCES}

1. Worldometer (2021) COVID-19 coronavirus pandemic.

2. Varma A, Dergaa I, Mohammed AR, Abubaker M, Al Naama A, et al. (2021) Covid-19 and diabetes in primary care- How do hematological parameters present in this cohort? Expert Review of Endocrinology \& Metabolism 16(3): 147-153.

3. Musa S, Dergaa I, Abdulmalik MA, Ammar A, Chamari K, et al. (2021) BNT162b2 COVID-19 Vaccine hesitancy among parents of 4023 young adolescents (12-15 Years) in Qatar. Vaccines 9(9): 981

4. Dergaa I, Abdelrahman H, Varma A, Yousfi N, Souissi A, et al. (2021) COVID-19 vaccination, herd immunity and the transition toward normalcy: challenges with the upcoming sports event. Annals of Applied Sport Science 9(3): 10.

5. Varma A, Dergaa I, Ashkanani M, Musa S, Zidan M (2021) Analysis of Qatar's successful public health policy in dealing with the Covid-19 pandemic. International Journal of Medical Reviews and Case Reports 5(2): 6-11

6. Dergaa I, Abubaker M, Souissi A, Abdul RM, Varma A, et al. (2022) Age and clinical signs as predictors of COVID-19 symptoms and cycle threshold value. Libyan Journal of Medicine 17(1): 2010337.

7. Trabelsi K, Ammar A, Masmoudi L, Boukhris O, Chtourou H, et al. (2021) Sleep quality and physical activity as predictors of mental wellbeing variance in older adults during COVID-19 lockdown: ECLB COVID-19 international online survey. International Journal of Environmental Research and Public Health 18(8): 4329

8. Trabelsi K, Ammar A, Masmoudi L, Boukhris O, Chtourou H, et al (2021) Globally altered sleep patterns and physical activity levels by confinement in 5056 individuals: ECLB COVID-19 international online survey. Biology of Sport, Vol. 38.

9. Dergaa I, Varma A, Tabben M, Malik RA, Sheik S, et al. (2021) Organising football matches with spectators during the COVID-19 pandemic: What can we learn from the Amir Cup Football Final of Qatar 2020? A call for action. Biology of Sport 38(4): 677-681.

10. Mohammed AR, Varma A, Abubaker M, Basheer SM, Manu L, et al. (2020) Should all patients having planned procedures or surgeries be tested for COVID-19? American Journal of Surgery and Clinical Case Reports 2(2): $1-3$.

11. Varma A, Dergaa I, Zidan M, Chaabane M (2020) Covid-19: "Drive thru swabbing hubs"- safe and effective testing for travellers. The Journal of Medical Research 6(6): 311-312

12. Varma A, Abubaker M, Dergaa I (2020) Extensive saliva based COVID-19 testing - the way forward to curtail the global pandemic? The Journal of Medical Research 6(6): 309-310

13. Musa S, Al Baker W, Al Muraikhi H, Nazareno D, Al Naama A, et al. (2021) Wellness program within primary health care: How to avoid "No Show" to planned appointments? - A patient-centred care perspective. Physical Activity and Health 5(1): 76-86.

14. Smith AC, Thomas E, Snoswell CL, Haydon H, Mehrotra A, et al. (2020) Telehealth for global emergencies: Implications for coronavirus disease 2019 (COVID-19). Journal of telemedicine and telecare 26(5): 309-313.

15. Kichloo A, Albosta M, Dettloff K, Wani F, El-Amir Z, et al. (2020) Telemedicine, the current COVID-19 pandemic and the future: a narrative review and perspectives moving forward in the USA. Family Medicine and Community Health 8(3): e000530.

16. Khairat S, Meng C, Xu Y, Edson B, Gianforcaro R (2020) Interpreting COVID-19 and virtual care trends: Cohort study. JMIR Public Health and Surveillance 6(2): e18811.
17. Gorodeski EZ, Goyal P, Cox ZL, Thibodeau JT, Reay RE, et al. (2020) Virtual visits for care of patients with heart failure in the era of COVID-19: A statement from the Heart Failure Society of America. Journal of Cardiac Failure 26(6): 448-456.

18. Srinivasan M, Asch S, Vilendrer S, Thomas SC, Bajra R, et al. (2020) Qualitative assessment of rapid system transformation to primary care video visits at an academic medical center. Annals of Internal Medicine 173(7): 527-535.

19. Wijesooriya NR, Mishra V, Brand PL, Rubin BK (2020) COVID-19 and telehealth, education, and research adaptations. Paediatric Respiratory Reviews 35: 38-42.

20. Donnelly C, Ashcroft R, Bobbette N, Mills C, Mofina A, et al. (2021) Interprofessional primary care during COVID-19: a survey of the provider perspective. BMC Family Practice 22(1): 31

21. Shankar M, Fischer M, Brown-Johnson CG, Safaeinili N, Haverfield MC, et al. (2020) Humanism in telemedicine: connecting through virtual visits during the COVID-19 pandemic. Ann Fam Med COVID Collect.

22. Bergman D, Bethell C, Gombojav N, Hassink S, Stange KC (2020) Physical distancing with social connectedness. The Annals of Family Medicine 18(3): 272-277.

23. Nouri S, Khoong E, Lyles C, Karliner L (2020) Addressing equity in telemedicine for chronic disease management during the Covid-19 pandemic. NEJM Catal Innov Care Deliv.

24. Crawford A, Serhal E (2020) Digital health equity and COVID-19: The innovation curve cannot reinforce the social gradient of health. Journal of Medical Internet Research 22(6): e19361.

25. Fujioka JK, Budhwani S, Thomas-Jacques T, De Vera K, Challa P, et al. (2020) Challenges and strategies for promoting health equity in virtual care: Protocol for a scoping review of reviews. JMIR Research Protocols 9(12): e22847.

26. Gomez T, Anaya YB, Shih KJ, Tarn DM (2021) A qualitative study of primary care physicians' experiences with telemedicine during COVID-19. The Journal of the American Board of Family Medicine 34(Supplement): S61-S70.

27. Contandriopoulos D, Brousselle A, Breton M, Duhoux A, Hudon C, et al Analyse des impacts de la rémunération des médecins sur leur pratique et la performance du système de santé au Québec.

28. Bourassa FM, Laberge M, Adisso L, Attisso E, Bourdeau-Chabot M, et al. (2021) Encadrement normatif et contractuel des pratiques de téléconsultation en clinique de première ligne. CIRANO.

29. Committee on Ethics in Science and Technology (2014) Clinical telehealth in Quebec: an ethical perspective.

30. Elgrably-Lévy N, Belzile G (2010) La télémédecine: améliorer le système de santé par l'innovation.

31. AMA digital health care 2016 \& 2019 study findings. American Medical Association.

32. Keesara S, Jonas A, Schulman K (2020) Covid-19 and health care's digital revolution. New England Journal of Medicine 382(23): e82.

33. Rogers EM (2010) Diffusion of innovations. Simon and Schuster

34. Murphy B (2021) How the telehealth boom is changing physician training. American Medical Association.

35. Van Galen LS, Wang CJ, Nanayakkara PWB, Paranjape K, Kramer MHH, et al. (2019) Telehealth requires expansion of physicians' communication competencies training. Medical teacher 41(6): 714-715. 